\title{
O trabalho e o estado de humor de funcionários de uma universidade pública
}

\author{
Work and mood among public university employees \\ El trabajo y el estado de humor de los funcionarios de una universidad pública
}

\author{
Felipe José Sousa Oliveira'; Letícia Yamawaka de Almeida"; Jaqueline Lemos de Oliveira"I"; \\ Lilian Carla de Almeida'v; Jacqueline de Souzav
}

\begin{abstract}
RESUMO
Objetivo: descrever as características do trabalho e o estado de humor de funcionários de uma Universidade Pública. Método: estudo quantitativo, transversal desenvolvido num campus universitário no interior do estado de São Paulo com 111 trabalhadores, aprovado pelo Comitê de Ética em Pesquisa sob o protocolo 2.170.360. Para a coleta de dados utilizou-se um questionário socioeconômico e Escala de Humor de BRUMS. Os dados foram analisados por meio de estatística descritiva. Resultados: A maioria dos participantes era de meia idade, trabalhavam nos setores administrativo/financeiro, com jornada de trabalho de 30 horas semanais e tempo de serviço até 10 anos. Identificaram-se altos níveis de humor positivo, sobretudo no fator vigor. Observou-se também níveis consideráveis dos estados emocionais ansioso e preocupado. Conclusão: embora a maioria dos participantes apresentasse altos níveis de humor positivo, identificou-se que cerca de $40 \%$ dos trabalhadores apresentaram altos escores nos itens relacionados à ansiedade e preocupação, que compõem o fator tensão da Escala de Humor de BRUMS
\end{abstract}

Descritores: Humor; fatores socioeconômicos; saúde mental; saúde do trabalhador.

\begin{abstract}
Objective: to describe the characteristics of work and mood among employees at a public university. Method: this quantitative, descriptive, cross-sectional study, conducted at a university campus with 111 employees in a city in São Paulo state, was approved by the research ethics committee (protocol 2.170.360). Data were collected by socioeconomic questionnaire and the Brunel Mood Scale, and were treated using descriptive statistics. Results: most of the participants were middle-aged, worked in administrative/financial positions, for 30 hours per week, and had worked there for up to 10 years. High levels of positive mood were identified, especially as regards the vigor factor. Levels of anxious and worried states of mind were also considerable. Conclusion: although most of the participants displayed high levels of positive humor, about $40 \%$ of the workers returned high scores on items relating to anxiety and worry, which make up the tension factor of the BRUMS mood scale.

Descriptors: Humor. socioeconomic factors. mental health. worker's health.
\end{abstract}

\section{RESUMEN}

Objetivo: describir las características del trabajo y el estado de humor de funcionarios de una Universidad Pública. Método: estudio cuantitativo y transversal, desarrollado en un campus universitario en el interior del estado de São Paulo junto a 111 trabajadores, aprobado por el Comité de Ética de Investigación bajo el protocolo 2.170.360. Para la recolección de datos, se utilizó un cuestionario socioeconómico y Escala de Humor de BRUMS. Los datos se analizaron por medio de estadística descriptiva. Resultados: La mayoría de los participantes era de mediana edad, trabajaba en los sectores administrativo/financiero, con jornada de trabajo de 30 horas semanales y tiempo trabajando de hasta 10 años. Se identificaron altos niveles de humor positivo, sobre todo en el factor vigor. Se observaron también niveles considerables de los estados emocionales ansioso y preocupado. Conclusión: aunque la mayoría de los participantes presentaba altos niveles de humor positivo, se identificó que cerca del $40 \%$ de los trabajadores presentó altos niveles en los ítems relacionados a la ansiedad y la preocupación, los cuales componen el factor tensión de la Escala de Humor de BRUMS.

Descriptores: Humor; factores socioeconómicos; salud mental; salud laboral.

\section{INTRODUÇÃO}

Com o avanço tecnológico e advento da globalização, observa-se um processo de significativas transformações no mundo do trabalho, em especial as novas exigências das formas de se trabalhar, que vislumbram a rapidez de produção ${ }^{1}$. No entanto, o ambiente de trabalho apresenta, por característica, um duplo papel, por um lado proporcionando o desenvolvimento dos indivíduos e o respectivo aumento da expectativa e da qualidade de vida quando exercido em um conjunto de condições adequadas e, por outro lado, favorecendo o desenvolvimento de doenças, podendo até culminar no encurtamento da vida ou na morte ${ }^{1}$.

'Enfermeiro. Escola de Enfermagem de Ribeirão Preto da Universidade de São Paulo. Brasil. E-mail: felipe.jose.oliveira@usp.br "Enfermeira. Doutoranda. Escola de Enfermagem de Ribeirão Preto da Universidade de São Paulo. Brasil. E-mail: leticia.almeida@gmail.com

'"Enfermeira. Doutoranda. Escola de Enfermagem de Ribeirão Preto da Universidade de São Paulo. Brasil. E-mail: jaquelemos@usp.br

IVEnfermeira. Mestra. Escola de Enfermagem de Ribeirão Preto da Universidade de São Paulo. Brasil. E-mail: lizarbrp@yahoo.com.br

vEnfermeira. Professora Doutora. Escola de Enfermagem de Ribeirão Preto da Universidade de São Paulo. Brasil. E-mail: jacsouza2003@usp.br 
Neste sentido, as complicações advindas da atividade ocupacional são influenciadas por fatores que caracterizam as rotinas às quais os funcionários estão submetidos, como aquelas que demandam maior aprimoramento, desempenho social e físico, a saber, a divisão de tarefas, cumprimento de carga horária, relacionamentos interpessoais e o gerenciamento de pessoal e das múltiplas atividades ${ }^{2}$.

A configuração de organizações trabalhistas ao longo dos séculos permitiu o desenvolvimento social e cognitivo da sociedade, reafirmando as máximas de responsabilidades e deveres. Porém, diante das novas tecnologias de comunicação, o profissional fica responsável por codificar e atender uma categoria maior de informações que, quando não gerenciadas adequadamente, causam um excesso de demandas, podendo influenciar a saúde mental, por meio da manifestação de insônia, fadiga, irritabilidade e outras queixas psíquicas ${ }^{3}$.

Nos últimos anos tem-se estudado aspectos inerentes à saúde mental dos trabalhadores devido aos altos níveis de absenteísmo, aposentadorias precoces e riscos à saúde associados à atividade profissional, no qual cada vez mais pessoas apresentam sofrimento emocional relacionado ao trabalho ou ocasionado por ele 4 .

O acúmulo de tarefas e o desenvolvimento de competências múltiplas, à medida em que evoluem, submetem os profissionais a situações de estresse, causando prejuízos no humor, influenciando negativamente na qualidade de vida, no uso de substâncias psicoativas, tais como o álcool e o tabaco, além de interferir nas atividades cotidianas, nas habilidades funcionais no ambiente de trabalho e, por conseguinte, na produtividade e nos índices de absenteísmo ${ }^{5}$.

Em relação à categoria de trabalhadores das universidades, destaca-se que o aumento dos cursos acadêmicos, das vagas disponibilizadas, da implementação de novas tecnologias e softwares têm exigido destes profissionais adaptações frequentes e maior qualificação para o atendimento de tais demandas suscetibilizando-os a estressores adicionais ${ }^{6,7}$.

Assim, entende-se que explorar aspectos psicológicos, sociais e emocionais desses trabalhadores possa fomentar o desenvolvimento de estratégias a serem incorporadas à gestão do trabalho, visando fortalecer os recursos adaptativos e de enfrentamento dos sujeitos frente aos fatores potencialmente estressores do ambiente laboral.

Portanto, o humor, caracterizado como uma experiência interna de como se encontra o tônus afetivo do sujeito em um dado momento, pode ser considerado como um relevante indicador do bem-estar psicológico ${ }^{8}$. Embora diversos autores tenham investigado o estado de humor nos mais diferentes agrupamentos de pessoas ${ }^{7-15}$, sobretudo entre atletas, nota-se uma lacuna na literatura acerca de estudos que investiguem o estado de humor dos trabalhadores em exercício, sinalizando a relevância do presente estudo.

Deste modo, o presente estudo teve como objetivo descrever as características do trabalho e o estado de humor de funcionários de uma universidade pública. Entende-se que os achados desta investigação poderão auxiliar no desenvolvimento de ações e diretrizes organizacionais voltadas à promoção de saúde mental do trabalhador, contribuindo para a melhoria dos níveis de saúde e bem-estar deste grupo.

\section{MEtodologia}

Trata-se de um estudo quantitativo, descritivo e de corte transversal desenvolvido no campus universitário de um município do interior do estado de São Paulo, que conta com oito unidades de ensino e sua prefeitura, responsável por desenvolver os serviços de infraestrutura física às demais unidades, além de promover atividades socioculturais e esportivas, de apoio ao ensino e pesquisa à comunidade local e externa ${ }^{16}$.

A população do estudo constituiu-se por trabalhadores das diferentes categoriais profissionais da prefeitura do campus, exceto os docentes. Os critérios de elegibilidade foram ser trabalhador concursado, não estar afastado das atividades de trabalho no período do estudo e não estar em período probatório. Compuseram a amostra de conveniência 111 trabalhadores que atendiam a tais critérios.

O período de coleta de dados foi de julho de 2017 a junho de 2018, realizada por uma enfermeira doutoranda e um aluno de graduação previamente treinados. Os trabalhadores foram abordados por meio do convite direto em sua unidade de trabalho e àqueles que atendiam aos critérios para a participação, procedeu-se a entrega de uma carta convite contendo o objetivo, o delineamento da pesquisa e o termo de consentimento livre esclarecido. Posteriormente, foram enviadas mensagens individualizadas, expedidas pelo pesquisador responsável, via endereço eletrônico aos trabalhadores previamente convidados para o estudo, que continham um breve comentário sobre o estudo e as orientações para que o participante acessasse o questionário na plataforma "GoogleDocs". Tendo recebido a comunicação da pesquisa, o participante preenchia o questionário e, ao término, as respostas eram gravadas numa base de dados comum a todos os participantes e armazenadas on-line. Ressalta-se que, para os trabalhadores que aceitaram participar da pesquisa, mas não tinham acesso à internet ou e-mail pessoal, o pesquisador responsável entregou os instrumentos impressos em suas respectivas unidades de trabalho. 
Os instrumentos utilizados para a coleta dos dados continham questões de cunho sociodemográfico, desenvolvidas pelos pesquisadores envolvidos no estudo, com base nos indicadores mínimos descritos pelo IBGE, contemplando aspectos relacionados à educação e condição de vida, família, cor ou raça e trabalho e a Escala de Humor de Brunel, BRUMS ${ }^{17-18}$. Tal escala consiste em uma adaptação do "Profile Mood States" (POMS), criada em 1971 por Mcnair, Lorr,e Droppleman ${ }^{17,18} \mathrm{com}$ a finalidade de observar os estados de flutuação do humor de pacientes psiquiátricos, por meio de 65 itens. Posteriormente, observou-se também a possibilidade de ampliação do uso deste instrumento para outras populações, sendo amplamente empregado em intervenções na psicologia do esporte ${ }^{19}$. Por se tratar de uma escala extensa e pela brevidade requerida durante a coleta de dados em pesquisas, diversas versões abreviadas foram desenvolvidas.

Neste sentido, a BRUMS tem se destacado devido ao seu rigoroso processo de validação, além de demonstrar a mesma eficácia da POMS. A validação da versão brasileira da BRUMS foi realizada por Rohlfs em um estudo com indivíduos atletas e não atletas, mostrando-se sensível e fidedigna na avaliação dos estados emocionais alterados de ambas as populações, com boa consistência interna, sendo os valores de alfa de Cronbach superiores a 0,70 para todas os construtos. Trata-se de um instrumento autoaplicável, de fácil compreensão e rápido preenchimento ${ }^{19,20}$.

O instrumento consiste num questionário estruturado com 24 itens, subdivididos em seis domínios: 1) Confusão: respostas à ansiedade/depressão, manifestados por sentimentos de incerteza, instabilidade para controle de emoções; 2) Depressão: remete-se a um estado depressivo, no qual a inadequação pessoal se faz presente. Indicação de humor deprimido e não depressão clínica; 3) Fadiga: estados de esgotamento, apatia e baixo nível de energia; 4) Raiva: relacionado a sentimentos de hostilidade. Estado emocional variando de sentimentos leves a estímulos do sistema nervoso autônomo; 5) Tensão: refere-se a alta tensão musculoesquelética, não observadas diretamente ou por manifestações psicomotoras; 6) Vigor: estados de energia, animação e atividade, indicando aspecto de humor positivo ${ }^{19}$.

Para o cálculo do escore, as opções de respostas são codificadas mediante uma escala Likert de cinco pontos (sendo 0 = nada e 4 = extremamente). Assim por meio da soma das pontuações específicas de cada item, obtém-se o escore que pode variar de 0 a 16 para cada domínio (estado de humor), quanto maior o valor do domínio, maior é a manifestação do respectivo estado de humor ${ }^{19}$.

Na fase da análise dos dados, as respostas dos participantes foram organizadas em Planilhas Google, o banco de dados consolidado foi transportado para o pacote estatístico Statistical Package for the Social Sciences (SPSS ${ }^{\circledR}$ ), versão 17.0, e os dados submetidos à estatística descritiva para definição das frequências, média, mediana e desvio-padrão.

Este estudo foi submetido à apreciação do Comitê de Ética em Pesquisa da Escola de Enfermagem de Ribeirão Preto-USP, sendo aprovado sob o protocolo 2.170.360. Ressalta-se que os aspectos éticos sobre a pesquisa envolvendo seres humanos foram contemplados, conforme a Resolução 466/12 do Conselho Nacional de Ética em Pesquisa.

\section{RESULTADOS}

$\mathrm{Na}$ amostra estudada identificou-se a prevalência do sexo masculino, faixa etária variou entre 27 e 70 anos ( $\mu$ : 47,4; dp: 9,86) e com renda familiar na faixa de 1.400 a 23.000 reais ( $\mu$ : 7.358 reais; dp: 4.629,48). A maioria se autodeclarou branca, residia em casa própria, era casada e possuía carro. A religião católica foi a mais referida. Em relação à escolaridade, observou-se que a maioria tinha o ensino médio ou superior completo (Tabela 1).

A tabela 1 apresenta, também, uma caracterização das atividades laborais dos participantes, considerando os aspectos: tipo de ocupação, tempo na ocupação atual e jornada semanal de trabalho.

Com relação ao tipo de ocupação, os trabalhadores foram divididos em quatro grupos, de acordo com o tipo de trabalho, a saber, o grupo administrativo/financeiro, que abarcou as atividades dos setores que desenvolviam atividades de segurança, guarda, vigilância, organização de materiais, compras, contabilidades e almoxarifado e, por fim, os serviços gerais e de pessoal. O segundo grupo, denominado de atendimento à comunidade, diz respeito aos setores relacionados ao serviço de promoção social, creche, moradia e atividades culturais, esportivas e informativas/publicitárias e o terceiro grupo, denominado de manutenção e operação, envolveu os serviços de apoio predial, hidráulica, elétrica e transporte. O quarto grupo, intitulado como apoio transitório, refere-se aos serviços de biotério, operacionalização, apoio técnico e bibliotecário.

Assim, verificou-se o predomínio do grupo administrativo/financeiro, com jornada de trabalho de 30 horas semanais ( $\mu: 15,1 ; d p: 11,3$; intervalo obtido 3 meses a 45 anos) e o tempo de 10 anos na ocupação atual ( $\mu$ de horas diárias 3,2; dp:0,5; intervalo obtido de 2 a 4 horas).

Em relação aos estados de humor, a tabela 2 apresenta a média do escore total de cada fator da escala de humor, com destaque para o fator vigor, referente aos estados de energia, animação e atividade, indicando humor positivo entre os participantes. Em contrapartida, os fatores confusão e depressão manifestaram-se com os menores níveis. 
Tabela 1: Perfil socioeconômico e descrição das atividades laborais dos participantes, Brasil, 2017.

\begin{tabular}{|c|c|}
\hline Variáveis & n (\%) \\
\hline \multicolumn{2}{|l|}{ Faixa etária } \\
\hline 27 a 40 anos & $32(28,8)$ \\
\hline 41 a 50 anos & $25(22,5)$ \\
\hline 51 a 70 anos & $48(43,2)$ \\
\hline Não informado & $06(5,4)$ \\
\hline \multicolumn{2}{|l|}{ Cor } \\
\hline Brancas /amarelas & $81(73,0)$ \\
\hline Pretas/Pardas & $28(25,2)$ \\
\hline \multicolumn{2}{|l|}{ Sexo } \\
\hline Feminino & $45(40,5)$ \\
\hline Masculino & $66(59,5)$ \\
\hline \multicolumn{2}{|l|}{ Estado civil } \\
\hline Casado & $82(73,9)$ \\
\hline Não casado & $25(22,5)$ \\
\hline Não informado & $4(3,6)$ \\
\hline \multicolumn{2}{|l|}{ Nível de Escolaridade } \\
\hline Ensino Fundamental & $11(9,9)$ \\
\hline Ensino Médio & $32(28,8)$ \\
\hline Nível Superior & $47(42,3)$ \\
\hline Pós-Graduação & $17(15,3)$ \\
\hline Não informado & $4(3,6)$ \\
\hline \multicolumn{2}{|l|}{ Religião } \\
\hline Católico & $63(56,8)$ \\
\hline Protestante & $18(16,2)$ \\
\hline Espírita & $14(12,6)$ \\
\hline Umbanda & $1(0,9)$ \\
\hline Sem religião & 0 \\
\hline Outra & $2(1,8)$ \\
\hline Não informado & $4(3,6)$ \\
\hline \multicolumn{2}{|l|}{ Moradia } \\
\hline Própria & $101(91,0)$ \\
\hline Alugada & $7(6,3)$ \\
\hline Outro & $3(2,7)$ \\
\hline \multicolumn{2}{|l|}{ Possuía carro } \\
\hline Sim & $105(94,6)$ \\
\hline Não & $6(5,4)$ \\
\hline \multicolumn{2}{|l|}{ Tipo de ocupação } \\
\hline Administrativo/financeiro & $43(38,7)$ \\
\hline Atendimento a comunidade & $36(32,4)$ \\
\hline Manutenção e operação & $19(17,1)$ \\
\hline Apoio transitório & $08(7,2)$ \\
\hline Não informado & $05(4,5)$ \\
\hline \multicolumn{2}{|l|}{ Tempo na ocupação } \\
\hline Até 10 anos & $46(41,4)$ \\
\hline De 11 a 20 anos & $26(23,4)$ \\
\hline De 21 a 30 anos & $23(20,7)$ \\
\hline Mais que 30 anos & $10(9,1)$ \\
\hline Não informado & $6(5,4)$ \\
\hline \multicolumn{2}{|l|}{ Jornada de trabalho/semana } \\
\hline Até 20 horas & $5(4,5)$ \\
\hline Até 30 horas & $81(73,0)$ \\
\hline Até 40 horas & $23(20,7)$ \\
\hline Não informado & $2(1,8)$ \\
\hline
\end{tabular}


Tabela 2: Escore médio dos domínios do humor de acordo com o tipo de ocupação, Brasil, 2017.

\begin{tabular}{|c|c|c|c|c|c|c|c|c|c|c|c|c|}
\hline \multirow{3}{*}{ Humor } & \multicolumn{12}{|c|}{ Tipo de ocupação } \\
\hline & \multicolumn{3}{|c|}{$\begin{array}{l}\text { Administrativo/ } \\
\text { financeiro }\end{array}$} & \multicolumn{3}{|c|}{$\begin{array}{l}\text { Atendimento à } \\
\text { comunidade }\end{array}$} & \multicolumn{3}{|c|}{ Manutenção e operação } & \multicolumn{3}{|c|}{ Apoio transitório } \\
\hline & Intervalo & $\mu$ & $d p$ & Intervalo & $\mu$ & $d p$ & Intervalo & $\mu$ & $d p$ & Intervalo & $\mu$ & $d p$ \\
\hline Vigor* & $3-15$ & 9,2 & 3,0 & $6-14$ & 9,8 & 2,3 & $0-14$ & 9,0 & 4,6 & $5-13$ & 9,5 & 2,4 \\
\hline Tensão & $0-15$ & 3,8 & 2,5 & $0-11$ & 4,8 & 3,0 & $0-8$ & 2,6 & 2,3 & $0-12$ & 3,6 & 4,3 \\
\hline Raiva & $0-11$ & 3,6 & 2,5 & $0-11$ & 4,1 & 2,9 & $0-7$ & 1,5 & 1,8 & $0-9$ & 3,4 & 3,2 \\
\hline Fadiga & $0-15$ & 4,4 & 3,9 & $0-14$ & 5,5 & 3,8 & $0-8$ & 1,8 & 2,4 & $0-13$ & 4,7 & 5,3 \\
\hline Confusão & $0-12$ & 2,0 & 2,7 & $0-7$ & 2,9 & 2,5 & $0-5$ & 1,1 & 1,4 & $0-10$ & 2,6 & 3,9 \\
\hline Depressão & $0-8$ & 1,8 & 2,2 & $0-11$ & 3,3 & 3,1 & $0-4$ & 0,5 & 1,2 & $0-11$ & 1,7 & 3,9 \\
\hline
\end{tabular}

*Único domínio relacionado ao humor positivo, quanto maior o escore, melhor o humor. $\mu$-média; dp - desvio padrão.

A Figura 1 apresenta a distribuição dos participantes, considerando o escore obtido nos 24 itens que compõem a escala de humor.

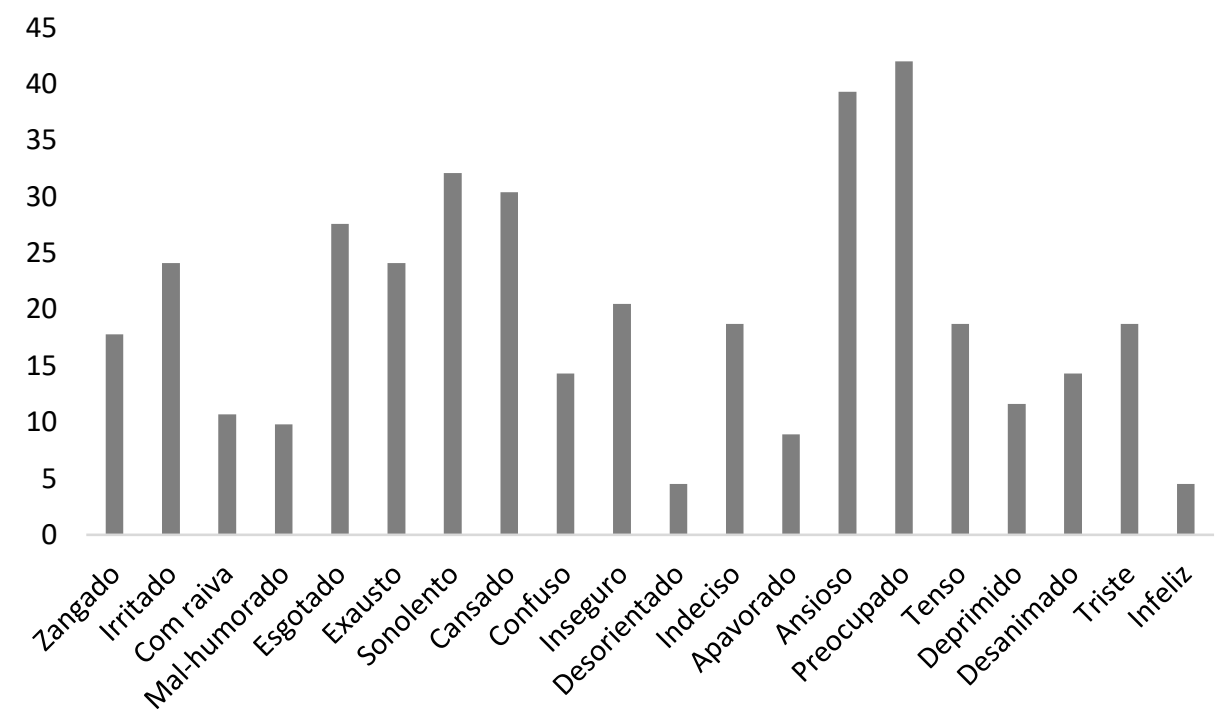

Figura 1: Distribuição dos participantes de acordo com os escores dos itens correspondentes ao humor negativo da Escala de Humor de BRUMS, Brasil, 2017.

Assim, embora a maioria dos participantes apresentasse altos níveis de humor positivo, identificou-se que cerca de $40 \%$ dos trabalhadores apresentaram altos escores nos itens relacionados à ansiedade e preocupação, que compõem o fator tensão da escala de humor de BRUMS.

\section{DISCUSSÃO}

O presente estudo teve por objetivo descrever as características do trabalho e o estado de humor de funcionários de uma universidade pública. Destarte, identificou-se que a maior parte dos trabalhadores exercia funções relacionadas ao trabalho administrativo/financeiro ou de atendimento à comunidade, estavam na ocupação atual, em média, há 15 anos, trabalhavam cerca de seis horas diárias e declararam renda familiar de aproximadamente R\$ 7.358,06.

No que se refere ao perfil socioeconômico, observou-se a prevalência do sexo masculino. Este achado, embora seja de uma amostra de conveniência, corresponde ao identificado em pesquisas prévias realizadas com trabalhadores de universidade ${ }^{21,22}$. Tal máxima confirma ainda os efeitos da divisão patriarcal que regia as relações sociais, na qual culturalmente aos homens era delegada a responsabilidade pela aquisição financeira que custearia os gastos familiares e às mulheres o cuidado do lar, educação e criação dos filhos ${ }^{23}$. 
Ademais, as mulheres exercem dupla jornada de trabalho, tanto na esfera doméstica como profissional, e isto pode ocasionar sobrecarga e cansaço físico, mental e, consequentemente, adoecimento ${ }^{22}$, podendo, quando não amenizada, desencadear distúrbios que acometem o bem-estar mental dessas trabalhadoras. Por essa razão, as licenças médicas relacionadas a questões de saúde mental, como os transtornos de humor, são maiores entre o público feminino $^{21,22}$. Deste modo, embora o presente estudo não tenha explorado as diferenças de gênero, cabe ressaltar que as especificidades deste grupo suscitam um olhar mais apurado em relação aos aspectos emocionais e seus desdobramentos.

No que concerne à faixa etária, observou-se que 43,2\% dos participantes deste estudo tinham entre 51 a 70 anos, demonstrando o envelhecimento da força de trabalho, tendência corroborada por outros estudos ${ }^{22-25}$. Segundo a literatura, o envelhecimento é um risco adicional para o desenvolvimento de problemas de saúde e incapacidade produtiva e, alicerçado por esta concepção, afirma-se que tais fatores podem dificultar a execução de determinadas tarefas, em especial aquelas que exigem maior desgaste físico. Diante disso, o trabalhador sente-se menos produtivo, apresenta maiores dificuldades na tomada de decisões, podendo seu bem-estar mental ser comprometido, afetando diretamente seu estado de humor e, consequentemente, tornando-se mais ausente no trabalho ${ }^{26}$. Por outro lado, a experiência a ser compartilhada por estes trabalhadores com os mais jovens deve ser considerada, pois quando bem administrada, tal troca pode ser decisiva em relação à produtividade no ambiente de trabalho.

Em relação às atividades laborais, o grupo administrativo/financeiro, em geral, possui cinco funções básicas: dirigir, atender, reunir, participar e concentrar, por isso fica, na maioria das vezes, restrito a computadores e a um ambiente único. Por esse motivo, a questão que mais afeta o trabalhador desta área é o desgaste mental, particularmente a fadiga e a tensão, uma vez que eles são responsáveis pela administração da pressão organizacional, de modo rápido e resolutivo ${ }^{27}$.

Considerando que o indivíduo dispende ao menos um terço do dia em sua jornada de trabalho, é importante garantir um ambiente laboral adequado para seu desenvolvimento pessoal e profissional, uma vez que o trabalho é um condicionante da qualidade de vida e estado de saúde. Trabalhadores submetidos a longas jornadas de trabalho podem apresentar estados de fadiga e tensão, decorrentes da necessidade de desempenhar uma função complexa, repetitiva e que exija atenção².

No tocante à amostra estudada, tanto o ambiente de trabalho quanto a jornada semanal não parecem se configurar como fonte de estresse para estes funcionários, nessa perspectiva, identificou-se que, em geral, os trabalhadores apresentavam o humor positivo, sobretudo no fator vigor, que analisa os estados de energia, animação, alerta e atividade. Estudos prévios sobre o estado de humor desenvolvidos com outras populações identificaram resultados semelhantes ${ }^{10-13}$. Entretanto, ressalta-se que tais investigações permaneceram restritas a atletas e as justificativas para estes perfis se dão especificamente por reações do contexto esportivo.

No presente estudo, provavelmente, as características sociodemográficas e laborais da amostra contribuíram para o estado de humor positivo identificado. Outrossim, aspectos referentes ao espaço físico e às relações no ambiente de trabalho podem ser potenciais mediadores de tais resultados. Outros fatores, tais quais a satisfação com o trabalho e o clima organizacional, podem influenciar nos estados de humor e nos demais âmbitos da saúde mental dos trabalhadores ${ }^{14,15}$.

Nesta perspectiva, entende-se que o estado de humor também pode estar relacionado a questões como divisão e distribuição de tarefas, as condições essenciais para o trabalho e o funcionamento psíquico dos servidores, ou seja, se o indivíduo encontra satisfação e valorização no trabalho, poderá se desenvolver profissionalmente com maior facilidade e isso certamente contribui para seu estado de humor ${ }^{28}$.

Destaca-se que um percentual importante de participantes apresentou altos escores nos itens relacionados à ansiedade e preocupação, mensurados pela escala de humor. Tal resultado pode indicar que, apesar do trabalhador apresentar bom humor, algumas exigências do contexto de trabalho ou pessoais geram tais sentimentos, os quais, a longo prazo, podem culminar em uma situação de sofrimento psíquico.

Ademais, cabe ressaltar as possíveis influências do atual cenário da educação e das universidades públicas brasileiras na saúde mental destes trabalhadores. Este cenário tem ocupado um importante espaço de debate no âmbito acadêmico e social com discursos fortemente incrementados pela mídia jornalística ${ }^{29-31}$.

Tanto em artigos acadêmicos quanto em algumas matérias de jornais discute-se que mesmo formando profissionais altamente qualificados e ocupando boas posições inclusive em rankings internacionais, tais instituições têm sido alvos de contingenciamentos e medidas que contribuem para a precarização do trabalho como o Programa de Incentivo à Demissão Voluntária (PIDV), a suspensão dos concursos públicos e dos reajustes salariais, a redução de carga horária e de salários, a terceirização de algumas funções e o discurso de privatização ${ }^{29-31}$. 
Tais autores referem que essas medidas compõem um processo de "ataque político ideológico" 30:627 com vistas à desqualificação dos servidores públicos. Destacam ainda que a mídia tem disseminado a ideia de que tais trabalhadores são altamente privilegiados, descompromissados e ineficientes ${ }^{29-31}$ sem contextualizar adequadamente a "função e especificidade" ${ }^{30: 629}$ do trabalho destes funcionários que é essencial para a execução efetiva das políticas públicas e sociais. Entende-se, portanto, que a ansiedade e preocupação apresentada pelos participantes do presente estudo seja influenciada por tais características deste cenário político-social.

Assim, intervenções de promoção de saúde focadas na redução e/ ou manutenção destes estados emocionais, bem como oferecimento de um maior repertório de estratégias de enfrentamento do estresse devem ser explorados no ambiente laboral, visando prevenir o sofrimento psíquico desta população.

Outro achado desta amostra é o tempo médio na ocupação atual (15 anos). Os trabalhadores que desempenham a mesma função por muitos anos podem desenvolver desgastes físicos e mentais, relacionados diretamente à execução contínua das mesmas atividades ${ }^{28}$. Tal perspectiva pode produzir efeitos no estado de humor dos mesmos, sobretudo no estado de fadiga, gerando esgotamento, apatia, baixos níveis de energia e consequentes reações negativas perante a execução dos afazeres.

A partir dos achados do presente estudo, entende-se que, apesar dos funcionários apresentarem o humor positivo, algumas características como as especificidades da função e o tempo de ocupação são aspectos a serem considerados em termos de saúde emocional, além disso, uma parcela notável dos trabalhadores mostrou-se ansiosa e preocupada.

Dessa forma, desenvolver atividades de promoção e manutenção da saúde mental no ambiente laboral pode ser um aspecto preventivo em termos de sofrimento psíquico, podendo contribuir para a qualidade do trabalho e para a valorização profissional. Esta recomendação corrobora estudo prévio cujos autores inferem acerca da busca de estratégias que estimulem o engajamento em atividades de promoção do bem-estar físico e mental dos funcionários por parte dos mesmos e de seus empregadores. Além do que, programas motivacionais e de incentivo para o exercício físico, para o convívio social e para o aprimoramento profissional são recomendados ${ }^{28}$.

As limitações do presente estudo constituíram-se no desenvolvimento do mesmo em apenas um campus universitário, bem como a amostra de conveniência, que restringe as possibilidades de generalização dos resultados. Ademais, a utilização de indicadores específicos de ansiedade ou instrumentos para triagem de transtornos mentais comuns poderia proporcionar resultados mais conclusivos em relação à saúde mental do grupo estudado.

\section{CONCLUSÃO}

Os resultados do estudo evidenciaram bons níveis do estado de humor positivo dos trabalhadores, sobretudo no tocante ao fator vigor. Não obstante, níveis consideráveis dos estados emocionais ansioso e preocupado foram verificados. Sugere-se que alguns aspectos das condições laborais desta amostra, tais quais os atrativos das carreiras públicas e a estabilidade profissional, possam influenciar os estados de humor destes indivíduos. Além disso, depreende-se que aspectos relacionados às mudanças político-sociais correntes possam estar contribuindo para o aumento da ansiedade e preocupação nesse grupo de trabalhadores.

Nesta perspectiva, entende-se que o planejamento de intervenções de promoção da saúde mental são ações importantes a serem desenvolvidas no ambiente laboral, visando a manutenção do estado de humor positivo e a redução dos níveis de ansiedade e preocupação, de forma a conduzir o crescimento pessoal e social, bem como ampliar o repertório de estratégias de enfrentamento das adversidades destes funcionários, contribuindo para a manutenção da saúde e do bem-estar dos mesmos.

\section{REFERÊNCIAS}

1. Sanches EN, Santos JDF. Stress among university health teachers: generating situations, symptoms and coping strategies. Psico. argum. [online]. 2013 [cited 2017 May 20]; 31(75). DOI: http://dx.doi.org/10.7213/psicol.argum.31.075.DS04

2. Costa LST, Monte PR, Possobon RF, Ambrosano LMB. Prevalence of Burnout Syndrome in a Sample of Brazilian University Professors. Psico. reflexão e crítica [online]. 2013 [cited 2017 May 20]; 26(4). DOI: http://dx.doi.org/10.1590/S010279722013000400003

3. Guirado GMP, Pereira NMP. Use of the Self-Reporting Questionnaire (SQR-20) for determination of physical and psychoemotional symptoms in employees of a metallurgical industry located at Vale do Paraíba - Sao Paulo state - Brazil. Cad. saúde coletiva [online]. 2016 [cited 2017 May 20]; 24(1):92-98. DOI: http://dx.doi.org/10.1590/1414-462X201600010103

4. Marques DO, Pereira MS, Souza ACS, Oliveira E. Absenteeism - illness of the nursing staff of a university hospital. Rev. bras. enferm. [Internet]. 2015 [cited 2017 May 20]; 68(5). DOI: http://dx.doi.org/10.1590/0034-7167.2015680516i

5. Oliveira LO, Baldaçara LR, Maia MZB. Absence from work due to mental disorders among federal government workers in Tocantins, Brazil. Rev. bras. saúde ocupa. [Internet]. 2015 [cited 2017 May 20]; 24(1). DOI: http://dx.doi.org/10.1590/03037657000092614 
6. Alarcon ACRS, Guimarães LAM. Prevalence of minor mental disorders among employees of a public university of the state of Mato Grosso Do Sul, Brazil. Rev. sulam. psico. [Internet]. 2016 [cited 2017 May 25]; 4(1). Available from:

http://www.revista.unisal.br/am/index.php/psico/article/view/106

7. Gavin RS, Reisdorfer E, Donato EC, Reis LN, Zanetti AC. Association between depression, stress, anxiety and alcohol use among civil servants. Rev. eletrônica saúde mental álcool drog. [Internet]. 2015 [cited 2017 May 26]; 11(1). DOI:

http://dx.doi.org/10.11606/issn.1806-6976.v11i1p2-9

8. Trevisan PR, Schwartz GM. Assessment of Mood States in the Royal Academy of Dance Examination System. Psic.: teor. e pesq. [Online]. 2016 [cited 2017 May 27]; 33(1). DOI: http://dx.doi.org/10.1590/0102.3772e3338

9. Ferreira TS, Moreira Z, Guo J, Noce F. Effects of a 12-hour shift on mood states and sleepiness of Neonatal Intensive Care Unit nurses. Rev. Esc. Enferm. USP. [Internet]. 2017 [cited 2017 May 27]; 51(1). DOI: https://doi.org/10.1590/s1980220x2016033203202

10. Brandt R, Liz Carla M, Crocetta TB, Arab C, Bevilacqua G, Dominski FH et al. Mental health and associated factors in athletes during the open games of Santa Catarina. Rev. bras. med. Esporte [Internet]. 2014 [cited 2017 May 27]; 20(4):276-280. DOI: http://dx.doi.org/10.1590/1517-86922014200401607

11. Brandt R, Ribeiro HN, Fernandes AR, Andrade A, Viana MS. Mood states of non-professional soccer referees. Rev. educ. fis. UEM. [Internet]. 2013 [cited 2017 May 27]; 23(4). Available from: http://www.scielo.br/scielo.php?script=sci_arttext\&pid=S1983-30832012000400007

12. Leite GS, Amaral DP, Oliveira RS, Oliveira Filho CW, Mello MT, Brandão MRF. Relationship between mood states, heart rate variability and creatine kinase of brazilian para-athletes. Rev. educ. fis. UEM. [Internet]. 2013 [cited 2017 May 27]; 24(1). Available from: http://www.scielo.br/scielo.php?script=sci_abstract\&pid=S1983$30832013000100004 \& \operatorname{lng}=e n \& n r m=i s o \& \operatorname{lng}=e n$

13. Rotta TM, Miranda IC, Oliveira WF. Applicability of Brums: mood profile in athletes of volleyball and tennis in high performance. Rev. bras. med. esporte. [Internet]. 2014 [cited 2017 May 30]; 20(6):424-428. DOI: http://dx.doi.org/10.1590/151786922014200602188

14. Rueda FM, Lima RC, Raad AJ. Quality of life and job satisfaction: relationship between scales that measure these constructs. Bol. psicol. [Internet]. 2014 [cited 2017 May 30]; 64(141). Available from: http://pepsic.bvsalud.org/scielo.php?script=sci_arttext\&pid=S0006-59432014000200003\&lng=pt\&tlng=pt

15. Silva DSD, Tavares NVS, Alexandre ARG, Freitas DA, Brêda MZ, Albuquerque MCS, Melo Neto VL. Depression and risk of suicide in professional Nursing: integrative review. Rev. Esc. Enferm. USP. [Internet]. 2015 [cited 2017 May 30]; 49(6). DOI: http://dx.doi.org/10.1590/S0080-623420150000600020

16. Universidade de São Paulo. Prefeitura do campus. Ribeirão Preto: USP. Available from: http://www.prefeiturarp.usp.br/

17. Terry PC, Lane AM, Fogarty GJ. Construct validity of the Profile of Mood States - Adolescents for use with adults. Psychol. Sport Exerc. [Internet]. 2003 [cited 2017 May 30]; 4(2). DOI: https://doi.org/10.1016/S1469-0292(01)00035-8

18. Macnair DM, Lorr M, Droppelman LF (1971). Manual for the Profile of Mood States (POMS). San Diego: Educational and Industrial Testing Service.

19. Rohlfs ICPM. Validação do teste de BRUMS para avaliação de humor em atletas e não atletas brasileiros [master thesis]. Florianópolis: Universidade do Estado de Santa Catarina; 2006. Available from: http://www.tede.udesc.br/handle/tede/992

20. Rohlfs ICPM, Rotta TM, Luft CDB, Andrade A, Krebs RJ, Carvalho T. Brunel Mood Scale (BRUMS): an instrument for early detection of overtraining syndrome. Rev. bras. med. esporte. [Internet]. 2008 [cited 2017 May 27]; 14(3). DOI: https://doi.org/10.1590/S1517-86922008000300003

21. Sousa LP, Guedes DR. A desigual divisão sexual do trabalho: um olhar sobre a última década. Estud. av. [Internet]. 2016 [cited 2017 May 22]; 30(87). DOI: http://dx.doi.org/10.1590/S0103-40142016.30870008

22. Batista I. Absenteísmo por licença médica em servidores de uma Instituição Federal de Ensino Superior em Minas Gerais [master thesis]. Uberlândia: Universidade Federal de Uberlândia; 2014. Available from: https://repositorio.ufu.br/handle/123456789/12822

23. Carrillo-García C, Solano-Ruíz MC, Martinez-Roche ME, Gomez-García Cl. Job satisfaction among health care workers: the role of gender and age. Rev. latinoam. enferm. [Internet]. 2013; [cited 2017 May 27]; 21(6). DOI: https://doi.org/10.1590/01041169.3224.2369

24. França IMS. Atividade laboral como contexto de sofrimento e adoecimento psíquico: análise dos servidores públicos em instituição federal brasileira de ensino superior [master thesis]. Natal: Universidade Federal do Rio Grande do Norte; 2016. Available from: https://repositorio.ufrn.br/jspui/handle/123456789/22358

25. Alves SB. Aging workers in brazil: reflections on the right to social protection in peripheral capitalism. Rev. cul. jur. [Internet]. 2015 [cited 2017 May 30]; 2(4). Available from: http://www.culturasjuridicas.uff.br/index.php/rcj/article/view/141

26. Figueira TG. Bem-estar, mal-estar e qualidade de vida no trabalho em uma instituição pública brasileira [doctoral dissertation]. Brasília: Universidade de Brasília; 2014. Available from: http://bdtd.ibict.br/vufind/Record/UNB_f09894f52c979337170326d434c353a2

27. Ferraz F, Hecksher S, Carvalho E. Presenteísmo: as perdas diárias e silenciosas. Congresso Nacional de Excelência em Gestão [internet]. 2016 [cited 2017 Jun 01]; 1-13. Available from: http://www.inovarse.org/sites/default/files/T16_188.pdf

28. Servino S, Rabelo E, Campos RP. Stress and coping strategies among information technology professionals. Rev. interinst. Psicol. [Internet]. 2013 [cited 2017 Oct 20]; 6(2). Available from: http://pepsic.bvsalud.org/scielo.php?script=sci_arttext\&pid=S1983$82202013000200007 \& \operatorname{lng}=p t$ 
29. Vanz SAS, Dominique AP, Lascurain Sánchez ML, Sanz Casado E. University rankings and the challenge for the Brazilian universities. Encontros Bibli: revista eletrônica de biblioteconomia e ciência da informação [Internet]. 2018 [cited 2019 Aug 06]; 23(53):39-51. DOI: http://dx.doi.org/10.5007/1518-2924.2018v23n53p39

30. Druck G; Filgueiras L; Moreira U. Tax adjustment and the Brazilian public universities: the new investement of the World Bank. Cadernos do CEAS: Revista crítica de humanidades. [Internet]. 2018 [cited 2019 Aug 06]; (242):602-634. Available from: https://cadernosdoceas.ucsal.br/index.php/cadernosdoceas/article/view/411

31. Gaspardo M. Desafios das universidades públicas brasileiras: gestão ou política? Brasil de Fato [Internet]. 2019 [cited 2019 Aug 06]. Available from: https://www.brasildefato.com.br/2019/01/24/artigo-or-desafios-das-universidades-publicas-brasileirasgestao-ou-politica/ 